\title{
Necessidades de Orientação Educativa Na Universidade Regional do Cariri
}

\author{
Irineide Beserra Braga ${ }^{1}$; Marcus Nascimento Coelho ${ }^{2}$
}

\begin{abstract}
Resumo: O presente trabalho investiga as necessidades de orientação dos alunos da Universidade Regional do Cariri - URCA, Crato, Ceará, oriundos dos cursos de direito, enfermagem e pedagogia. Tem por objetivo observar como percebem, eles próprios, suas necessidades de orientação; de que maneira crêem serem conscientes de sua importância e se saberiam como obter orientação em caso de necessidade. Também visa comparar se as necessidades de orientação são uma questão de gênero, se o curso tem alguma influência nas necessidades e se são diferentes as necessidades dos alunos primeiranistas, dos alunos do último ano de curso. As informações foram colhidas através de questionário utilizado para a avaliação das necessidades, especialmente desenvolvido para este fim. Os resultados desta pesquisa revelaram claramente que os alunos necessitam de orientação nas diversas áreas investigadas. A orientação profissional e a orientação acadêmica, representam uma necessidade para $86,84 \%$ e 79,64\% dos alunos, respectivamente. Além destas constatações a pesquisa revelou que os alunos dos primeiros anos de curso sentem mais necessidade de orientação acadêmica, enquanto os alunos em final de curso sentem mais necessidade de orientação profissional e ainda, que as mulheres tem mais necessidades de orientação, lhe conferem maior importância e, informaram maiores dificuldades em obter os serviços de que necessitam. Os resultados desta pesquisa também indicam a existência de uma demanda pela criação de um serviço de orientação universitária. A qualidade da educação superior, dentro de uma perspectiva orientadora, não se identifica somente com uma melhor preparação intelectual e técnica dos futuros graduados, mas com o favorecimento do processo de amadurecimento pessoal, que permita tomadas de decisões mais congruentes; capacitação para enfrentar situações problemáticas de forma mais adequada, bem como o desenvolvimento de uma personalidade equilibrada, que permita atuar com mais eficácia na sociedade e nas relações interpessoais. Estes são fatores suficientes para justificar uma intervenção orientadora nesta direção.
\end{abstract}

Palavras-chave: Necessidades, Orientação, Universidade

\section{Educational Needs Guidance in Regional University of Cariri}

\begin{abstract}
The present work investigates the needs for orientation of the students in the Cariri Regional University-URCA, Crato, Ceará, proceeding from the Law, Nursing and Pedagogy courses. The objective is to observe how they perceive their needs of orientation, how they are aware of the importance it represents to them, and if they would know how to get orientation if they needed to. The work also aims to compare whether the necessities of orientation are a matter of gender, if the course exerts any influence in the needs and whether the necessities of the freshmen students are different from the needs of the senior students of their courses. The informations were collected through a questionnaire used to evaluate the needs, specially developed for this purpose. The results of this research clearly revealed that the students need orientation in the several areas investigated. The professional and academic guidance, represent a necessity to $86,84 \%$ and $79,64 \%$ of the students, respectively. Beyond those evidences the research revealed that the freshmen students of the courses feel a greater need for academic orientation, while the senior students feel a greater need for professional orientation, and still, that the women feel a greater need for orientation, they give more importance to it, and they informed that have more difficulties to get the services they need. The results of this research indicate clearly the existence of a demand for the creation of an orientation service program in the University. The quality of the
\end{abstract}

\footnotetext{
${ }^{1}$ Psicóloga pela Faculdade Leão Sampaio - CE. Mestre em Ciências da Educação pela Faculdade Unisaber - Goiás.

E-mail: irineidebraga@msn.com;

${ }^{2}$ Doutor em Psicanálise pela Sociedade Internacional de Psicanálise de São Paulo. E-mail: marcnascoelho@bol.com.br.
} 
Undergraduate education, within an orientated perspective isn't only identified with a better intellectual and technical preparation of the future graduated students, but also with the fostering of the their maturing process, which will allow them to take more consistent decisions, make them able to face problematical situations, as well as to develop a level headed personality, that allows them to act with a greater efficacy in the society and in the inter-personal relations. Therefore, There are enough factors to justify an oriented intervention in this direction.

Keywords: Requirements, Guidance, University

\section{Introdução}

A orientação, não pretende, nem direta nem indiretamente, criar modelos de explicações para a conduta humana. Sua pretensão é muito mais de natureza prática. Pretende encontrar a melhor maneira de ajudar o indivíduo, em seu processo de desenvolvimento, cada vez que intervém num conjunto de elementos de um sistema, em que este encontra-se imerso.

A orientação eficaz precisa de grande quantidade de informação, elemento básico em todo processo Orientador. Porém, não é só isso. Segundo Echeverría (1991), a maioria dos pais e professores e até alguns orientadores, crêem que desenvolver um bom programa de orientação consiste em dar muitos dados informativos, seja mediante palestras ou através de apostilas ou bases de dados informatizados. Porém se observarmos o funcionamento de nossa sociedade poderemos ver que dispor de grande quantidade de dados não significa que as pessoas estejam melhor informadas. Em uma sociedade onde os meios de comunicação permitem acesso, quase instantaneamente, a maioria dos dados sobre qualquer matéria, os adultos e especialmente os jovens estão mais desinformados do que nunca. Dispor de grande quantidade de dados não é o mesmo que processar informação.

Segundo Katz (1988: 546), as funções da Orientação no processamento da informação são: ajudar os estudantes a selecionar a informação que possa ser importante para eleger uma carreira; zelar para que a dita informação seja o mais exata, completa e pertinente possível; facilitar o acesso a esta informação e assessorar os estudantes, para que a empreguem de maneira razoável. Porém, sendo esta a função orientadora básica no processo, sem os objetivos prévios e posteriores a esse processamento da informação, não poderíamos denominá-la Orientação, senão simplesmente de informação.

Para Watts (1988: 503), sobre os objetivos de um dos tipos de orientação. A Orientação vocacional, que são quatro: 1 . a consciência de si mesmo, que implica em valorar que tipo de pessoa se é, quais são as capacidades, interesses, valores, atitudes, porém que tipo de pessoa/profissional pretende ser; 2. a consciência das distintas oportunidades, não só no mundo laboral remunerado, senão em toda a gama possível de atividades a realizar, ao longo da vida, tal como descreve Super (1981) em "Arco de Carreras Vitalicias"; 3. aprender a tomar decisões, explorando os diferentes estilos de tomada de decisões, gerando alternativas no processo de recolhida de informações e 4. preparar para a

39 Id en lime Revista de Psicologia. Ano 9, No. 25, Fevereiro/2015 - ISSN 1981-1179. Edição eletrônica em http://idonline.emnuvens.com.br/id 
transição, não somente tomar a decisão, mas levá-la à prática. Este objetivo envolve questões concretas: preparar-se para uma entrevista, aprender a completar formulários etc. Também se refere a forma de enfrentar os períodos de transição.

Ainda segundo o mesmo autor, a informação é parte importante para poder

desenvolver estes objetivos, porém não suficiente. Um serviço de Orientação baseado somente na informação fundamenta-se em que: os alunos estão motivados para utilizar a informação, são capazes de entendê-la e podem relacioná-la com suas necessidades. Se um estudante cumpre estes pressupostos, não precisaria de nenhum programa de Orientação educativa, posto que sua maturidade pessoal lhe permitiria a tomada de decisões com total segurança.

A orientação tem como finalidade que o aluno alcance a plena realização pessoal em todas as dimensões (Rodriguez Espinar, 1985: 145). Sempre é um processo de desenvolvimento pessoal, educativo e social, não uma decisão tomada em função de uma informação recebida, senão uma série de decisões tomada em um tempo, que muitas vezes leva alguns anos. Portanto, Orientar, em educação, não é somente oferecer aos alunos grande quantidade de dados informativos, senão oferecer situações de aprendizagem onde possam obter experiência de sua própria subjetividade, dos valores e interesses que impõem os objetivos que pretende alcançar. É intentar que investiguem o sentido profundo que determina suas decisões e ações. É promover que a pessoa se interrogue sobre seu futuro, buscando um equilíbrio harmonioso entre suas necessidades e suas possibilidades. É uma formação.

Da palavra Orientação se pode advir diversos significados, porém não existe um acordo acerca do significado preciso (Tyler, 1983: 28). Muitos autores se referem a ela sem nenhum qualificativo que o acompanhe, senão simplesmente como Orientação. (Bisquerra, 1991).

A Orientação Vocacional, termo utilizado pela primeira vez em 1908 por Parsons, surge como uma preocupação social e educativa. Os indivíduos reclamam uma ajuda que lhe permita minimizar suas necessidades (Alvarez, 1992: 6). Esta concepção nasceu no âmbito extra-escolar, por conta da necessidade e tendência a integrá-la no processo escolar. Por essa razão alguns autores não encontram diferenças significativas entre Orientação Vocacional e Orientação Educativa, sobretudo aqueles que consideram a Orientação como um processo que se desenrola ao longo de toda vida (Orientação para a carreira). Outros autores como Rivas (1988: 30), consideram que:

A orientação chamada educativa tem outra justificação e outro sentido. Aí existe um problema relacionado com a aprendizagem escolar que afeta uma pessoa concreta, com respeito a dificuldade de alcançar um rendimento adequado (...).

De acordo com esta perspectiva, há uma significativa diferença entre a Orientação para a eleição profissional e ocupação, de todos os outros processos em que o aluno vai aprendendo no mundo educativo. Se quisermos manter a dupla denominação chegaríamos a um grave dilema:

40 Id en lime Revista de Psicologia. Ano 9, No. 25, Fevereiro/2015 - ISSN 1981-1179. Edição eletrônica em http://idonline.emnuvens.com.br/id 
designar a Orientação que recebem as pessoas em busca de um trabalho; após algum tempo desempregadas; ou quando desaparece o seu posto de trabalho, com sua ocupação como tal.

O contexto sócio-ambiental, quando nasceu esta denominação é radicalmente distinto do panorama atual. A eleição vocacional implicava a pessoa em um campo ocupacional fixo, onde haviam poucas possibilidades de trocar de ocupação, senão para melhorar seu posto de trabalho em um mesmo grupo ocupacional. Atualmente o contexto laboral é bastante diversificado, posto que exercer uma ocupação não é garantia que possam desempenhá-la por toda a vida laboral, seja por extinção da mesma ou por necessidade de aprender novas habilidades para desenvolvê-la.

A eleição vocacional já não se efetua uma vez na vida, senão provavelmente diversas vezes. Já não está condicionada a adolescência, havendo necessidade de trocas nas concepções orientadoras. O orientador agora, deve oferecer sua ajuda tanto no trâmite educativo que compõe a formação inicial, como nos diversos momentos em que a pessoa deve trocar de ocupação, de profissão, ou necessite atualizar sua formação para poder desempenhá-la adequadamente. Entra aqui a preocupação com a Orientação para a formação, de forma permanente, quase nunca levada a cabo.

Ulla Kann (1988) acredita que exercer a função de Orientador profissional é dispor de um poder de influência notável sobre as pessoas. Esta possibilidade de ascendência sobre os orientandos pode enforcar-se desde os diversos pontos de vista. Pode-se promover trocas sociais ou orientar para o controle social; pode-se intentar que a pessoa exerça sua liberdade ou que "aprenda" a alienar-se, pode-se orientar para o desenvolvimento de um espírito crítico construtivo ou para que desenvolva uma atitude resignada, conformista; pode-se promover a auto-realização ou a "domesticação".

Ao tratar esse tema, a autora define "transformação" e "domesticação", do seguinte modo:

Para mim, transformação significa liberar o indivíduo das opções profissionais tradicionais, não só no que diz respeito ao sexo, senão também a classe social, a raça, etc. A domesticação representa o reverso da medalha. Neste caso trata-se de fazer crer aos estudantes, que a melhor opção é conformar-se, ou pelo menos trata-se de alienar as pessoas a escolhas tradicionais. Por exemplo, não se pretende que mulheres aspirem a um emprego de tempo integral, seria melhor para essas uma atividade que possam deixar facilmente para casar-se, ter filhos e dedicar-se a outras tarefas familiares (ULLA KANN, 1988: 510).

A Orientação pode, no que se refere a tomada de decisões, utilizar-se para perpetuar desigualdades sociais ou contribuir para a igualdade de oportunidades, mediante a verdadeira educação compensatória. Acontece quando o orientador perde de vista a finalidade essencial de sua ação: que as pessoas se auto-realizem. Auto-realizar-se pressupõe poder desenvolver ao máximo suas capacidades, sem mais limitações do que aquelas que se impõem a cada pessoa, e que cada um, livremente as aceite. Esse axioma que parte da filosofia existencialista, implica conhecer o entorno 
social e constatar as limitações que este realmente impõe, sempre observando-se com uma nova visão, de forma a criar uma relação nova com a sociedade.

Segundo Kann (1988), citando a Watts e Herr (1997), existem quatro enfoques distintos do objetivo sócio-político implícito da Orientação Vocacional: 1. instrumento de controle social, que serve para adaptar os indivíduos às oportunidades de trabalho que realmente se lhes oferecem; 2. instrumento de troca social; 3. instrumento de troca individual e 4. se propõe a conscientizar os estudantes de toda a gama de oportunidades existentes e ajudar-lhes a eleger, com maior autonomia, a opção que mais se ajuste a suas necessidades e preferências.

Alguns concebem a orientação educativa e vocacional como um modelo pontual, para resolver problemas com a eleição da profissão ou ocupação. Estas concepções (Rivas: 1988) a princípio se assemelham ao modelo psicológico e clínico, da qual tomam suas características essenciais. Um exemplo representativo desta concepção poderia ser as características do modelo que propõe Rivas (1988: 30):
a) existe uma pessoa que apresenta um problema de decisão entre alternativas.
b) liberdade para solicitar ajuda
c) ser um processo de intercâmbio e exposição de informações relevantes para um problema concreto
d) existe um planejamento técnico de ações a realizar.
e) é dirigido e estruturado por profissionais.
f) a responsabilidade de assessoria revela-se na qualidade da informação ministrada.
g) é responsabilidade exclusiva do assessorado por em prática o projeto de tomada de decisões.

Baseado neste modo de entender o enfoque orientador, estão as concepções que consideram a Orientação como um processo que tem lugar nas diversas etapas evolutivas, haja visto o que nos diz Hoyt et all (1972):

\begin{abstract}
A educação para a carreira é a totalidade dos esforços da comunidade e do sistema educativo, para ajudar a todos os indivíduos a familiarizar-se com os valores de uma sociedade orientada para o trabalho, a integrar esses valores em sua estrutura pessoal de valores e colocá-los em prática em suas vidas, de tal maneira que o trabalho, seja possível, significativo e satisfatório para cada indivíduo.
\end{abstract}

Esta investigação parte da concepção de que a Orientação pode ser uma importante ajuda em momentos pontuais, porém envolve muito mais que isso. Inclui a possibilidade de relacionar o programa escolar com as necessidades do aluno, prolongar a Orientação para além do currículo escolar e preparar estes para as mudanças aceleradas da sociedade. Aceitando a educação para a 
carreira como enfoque essencial na Orientação, continuamos diante de um novo dilema: Orientação integrada no currículo ou Orientação com um currículo próprio?

Segundo García Hoz (1970: 243), a finalidade da educação personalizada é: "A capacitação de um sujeito para que seja capaz de formular e realizar o seu projeto pessoal de vida". Entende a Orientação, como pilar fundamental desta educação personalizada, é um processo individual, diferente para cada pessoa.

Segundo Herrera (1976) A orientação é uma espécie de processo necessariamente individual, que se realiza em cada sujeito, de acordo com suas características, seus problemas e suas necessidades. Afirma ainda que,

O papel do professor, tutor ou orientador, ante um grupo de estudantes, será oferecer ajuda para auxiliar na tomada de decisão dos orientandos, proporcionando o desenvolvimento máximo das habilidades necessárias para enfrentar qualquer situação escolar, pessoal ou profissional. Para oferecer essa ajuda, se pode utilizar diversos enfoques, desde os diretivos aos não-diretivos. Pode fazer em forma de mandado, conselho, sugestão ou simplesmente através de informação (HERRERA, 1976: 14).

Segundo as características do tipo de relação que se estabeleça entre o orientando e o orientador, pode-se desenvolver dependência ou se promover a auto- orientação. Para que a Orientação seja verdadeiramente individualizada e eficiente, deve tender a promoção da autoorientação. Isso implica que a finalidade seja a autonomia da pessoa. Para que seja possível, deverá cuidar-se do tipo de relação e de comunicação entre as pessoas. Neste sentido, os autores Carkuff e Berenson (1967: 4) manifestam-se, ao dizer que, da relação significativa entre pessoas, seja entre professor-aluno, ou pai-filho, as conseqüências podem ser altamente construtivas ou deteriorantes, tanto no aspecto intelectual, como no psicológico.

Segundo os autores, algumas das características que deveriam nortear as ações orientadoras como a relação empática, posto que a comunicação verdadeira só pode dar-se em função da aceitação do outro, da compreensão de si e da abertura afetiva que esta provoca. A compreensão empática dos sentimentos e dos problemas do orientando é considerada um aspecto integral da Orientação, na maior parte das escolas. Assim, o confirmam as investigações sobre o fenômeno empático realizadas desde diversos enfoques" (Repetto, 1977: 200); também uma atitude de respeito a singularidade de cada sujeito, tendo em vista que desenvolveria a autonomia e a liberdade pessoal, só é possível desde que haja respeito ao modo de ver, sentir e atuar de cada pessoa. "O objetivo da orientação não consiste em internalizar um sistema de valores prefixados em cada indivíduo, senão que cada um integre uma estrutura de enfrentamento de si mesmo e de sua realidade e que atue com respeito a decisão tomada, seja ela qual for”. (Lázaro e Asensi, 1987:20); Ainda poderíamos considerar a implicação do sujeito em seu próprio orientador, uma vez que a informação que o orientador obtém do orientando, sobre sua 
personalidade ou sobre sua realidade social, somente serão eficazes se postas nas mãos do estudante, para que o mesmo possa manejá-la e utilizá-la em seu próprio benefício. Destacar a responsabilidade que o orientando tem em seu próprio processo, nos parece o caminho mais apropriado para fomentar sua autonomia.

\section{Conceituação de necessidades}

Não são poucos os estudos que há bastante tempo vem se preocupando com a conceituação do termo "necessidade". "Necessidade, num contexto de análise de necessidades significa uma discrepância ou espaço existente entre uma condição desejada ou aceitável e uma condição real, observada ou percebida" (Anderson y Ball, 1978). Como conseqüência, uma análise de necessidades é “(...) uma análise formal que mostra e documenta as lacunas e espaços existentes entre os resultados que se desejam alcançar, ordena essas lacunas (necessidades) em uma ordem prioritária...” (Kaufman, 1982: 75).

Tejedor (1990: 16) assinala quatro perspectivas em que se pode analisar a noção de necessidade: a perspectiva de discrepância: segundo a qual se entenderia por necessidade a diferença entre os resultados desejáveis e os observados; a perspectiva democrática: de acordo com a que concebe a necessidade como uma troca desejada pela maioria do grupo de referência; a perspectiva analítica: concebendo por necessidade a direção em que, segundo a informação disponível, se pode produzir uma melhora e a perspectiva diagnóstica: a que concebe uma necessidade como algo cuja ausência ou deficiência é prejudicial.

Entre os autores observa-se pouca diferenciação, com relação ao conceito de "necessidade". Entende-se portanto, a noção de "necessidade" como: a) algo que precisa ser complementado, pela escassez ou pela falta; b) Campanero Pérez (1992), consegue sintetizar esquematicamente os elementos fundamentais que definem uma análise de necessidades ${ }^{1}$. Necessidade como: Estudo sistemático antes de intervir; c) Esforço sistemático para identificar e compreender um problema; d) Estabelece a importância de um problema ou necessidade em relação com outros; e) Esforço por conseguir êxito na implantação de um novo programa; f) Utiliza fontes de informações sobre situações atuais e desejáveis; g) Provém de uma base para gerar as soluções ( ${ }^{1}$ Fonte: Pérez, C., Rivas, F. e Rocabert, E., Sistema de Assessoramento Vocacional para Ciegos, Revista de Psicologia General e Aplicada, Granada, Outubro, 1992).

Nota-se portanto um esforço na análise da necessidade, de uma forma organizada levando-se em conta a promoção de uma consciência crítica a respeito das condições atuais. 
Somente um levantamento seguido de análise criteriosa pode levar-se a identificar situações deficitárias ou insatisfatórias (necessidades) que afetam a um coletivo determinado e justificar a decisão de levar a cabo, qualquer intervenção no sentido de busca de melhoras.

\section{Justificativa}

A escolha da profissão é uma necessidade. A cada dia que passa vê-se que os jovens têm maior dificuldade para fazer suas opções. Assistimos a um final de século caracterizado por profundas transformações tecnológicas (Toffler, 1971, 1980, 1990), como consequiência um universo de cursos e novas especializações tem surgido. A tecnologia está presente em todas as áreas, e o fascínio por conhecer coisas novas vai tomando conta do jovem. Em geral o momento da escolha de uma profissão coincide com a fase do desenvolvimento na qual o jovem está se descobrindo novamente. Em meio a esse mundo de descobertas, ele faz a escolha possível no momento, sem ter muita consciência das influências que sofre e, principalmente, sem ter informações suficientes sobre a profissão que está escolhendo (Hamez, 1997: 10-13).

A complexidade sócio-econômica das últimas décadas tem revelado, de maneira inquestionável a necessidade da orientação educativa, como elemento essencial do sistema escolar e acadêmico, o que contribui para a melhoria da qualidade e para uma maior efetividade do ensino.

Segundo Lucchiari (1993) a análise de alguns relatórios de exames vestibulares de universidades públicas, como UFRS e a UFSC, constata que 25 a $30 \%$ dos alunos aprovados anualmente nos vestibulares declaram já ter iniciado antes um curso superior. Sabe-se também que é elevado o número de alunos que desistem, trocam de curso e solicitam transferências internas. Comparando o número de alunos que ingressam anualmente em cursos superiores, observa-se que 40 a $50 \%$ não chegam a concluir seus cursos.

A orientação para o desenvolvimento da carreira, também denominada de "orientação profissional", é uma das principais áreas de intervenção da orientação. Historicamente a orientação vem experimentado, conceitualmente, profundas transformações. "No passado se considerava a orientação profissional como uma intervenção que se reduzia a momentos pontuais, em que o indivíduo tinha que fazer uma eleição vocacional, à partir da qual, se daria todo o desenvolvimento de sua carreira profissional" (Alzina, 1998: 233-234).

A orientação é encarada neste trabalho como orientação geral, isto é, como um conjunto de princípios e técnicas que considera a pessoa a orientar, em sua personalidade integral, o que significa a coordenação de informes sobre o indivíduo e auxílio a este em diferentes áreas, de forma a torná-lo, pelo conhecimento de si mesmo, capaz de efetuar bons ajustamentos. 
O imenso campo das oportunidades de trabalho, de estudos, de recreação e de lazer, existentes na vida moderna criou, para o homem e, sobretudo para os adolescentes, um grande número de problemas que são sobretudo, de escolha de escolas, cursos, profissões, de amigos, de esposo ou de esposa e termina-se com a escolha do que fazer nos últimos anos de vida, de forma que nestes, possamos nos sentir realizados. Evidentemente, não seria este o quadro de uma sociedade de séculos passados, na qual os caminhos já se encontravam mais ou menos delineados para o indivíduo, desde o nascimento. $\mathrm{O}$ avanço da ciência e o progresso generalizado criaram novas oportunidades e estas deram origem a um crescente número de escolhas e de novos ajustamentos.

Jones (1951: 71-79) cita entre outras, várias alterações na estrutura social que permitem julgar da importância da orientação: 1. alterações na vida do lar: com o correr do tempo, menos oportunidades têm os pais de orientar seus filhos. A concorrência do cinema, dos clubes, das associações, escolas, e a falta de atividades caseiras, tendem a diminuir a importância do lar na educação e no encaminhamento dos jovens; 2. alterações nas condições de trabalho: a divisão crescente do trabalho; as especializações; as super- especializações profissionais; as ocupações; as atividades qualificadas, semi-qualificadas e não qualificadas. A rápida evolução das técnicas de trabalho cria problemas sérios de escolha de profissão, de ensino, de treinamento e de exercício profissional; 3. alterações na vida escolar: a escola procura acompanhar a evolução social mas não o faz no tempo certo. A população aumenta e o fenômeno da democratização da cultura vai tomando corpo; 4. alterações populacionais: o aumento da população é seguido de um menor confinamento nacional ou regional. As comunicações superam as distâncias e os povos se aproximam mais entre si, através do surgimento de maior migração.

O problema como se vê, não reside, pois, apenas em suplementar a deficiência de informes, a atuação de professores ou de pais, mas também, de encontrar meios pelos quais o adolescente possa, mediante auxílio externo, ajustar-se aos elementos dessa sociedade variada e complexa em que vive. A presente pesquisa busca investigar as necessidades de Orientação dos alunos da Universidade Regional do Cariri - URCA, que possam impedir a eficaz trajetória estudantil do jovem universitário.

A presente investigação revela-se importante nesta universidade, tendo em vista o grande número de solicitações, para migrações de alunos entre cursos, bem como das altas taxas de desistência. Também pelo fato de não haver nenhum Programa Específico de Orientação Educacional nesta Instituição de Nível Superior.

Trabalhou com os cursos de Direito, Enfermagem e Pedagogia, tendo em vista serem os dois primeiros, os cursos de maior procura pelos alunos e o último um dos cursos com menor taxa de concorrência. Também porque os mesmos refletem a realidade de Centros diferentes. Enfermagem pertence ao Centro de Ciências da Saúde, enquanto os cursos de Pedagogia e Direito pertencem ao Centro de Estudos Sociais Aplicados. As ações desenvolvidas nesta pesquisa fazem parte do Plano 
Estratégico da Fundação Universidade Regional do Cariri - URCA que, dentre outras, visa disseminar o conhecimento, além de ampliar o campo de atuação dos docentes/pesquisadores, através da produção científica. Não só como veículo de apresentação e divulgação dos Projetos, mas também porque sua realização representa uma contribuição significativa, na medida em que se consegue obter um impacto positivo na comunidade.

Nosso principal objetivo é identificar as Necessidades de Orientação Educativa, do ponto de vista da percepção do próprio estudante, em seu processo de inserção acadêmica, numa amostra representativa da população universitária da URCA, dos cursos de Pedagogia, Direito e Enfermagem, para que no futuro, estas informações, possibilitem a elaboração de programas de orientação educativa para os discentes.

Ante tal situação, nos perguntamos quais seriam as Necessidades de Orientação dos alunos da Universidade Regional do Cariri - URCA, que poderiam, caso sanadas a tempo, contribuir para resolver seus problemas reais, enquanto estudantes, futuros profissionais?

Dentro desta perspectiva o objetivo geral do presente estudo foi: analisar as necessidades de orientação educativa, do ponto de vista da percepção do próprio estudante, em seu processo de inserção acadêmica.

\section{Método}

Trata-se de um estudo de campo de natureza descritiva, cujas variáveis utilizadas são de natureza quantitativa e qualitativa. A amostra foi aleatória estratificada, onde os estratos foram os cursos de Direito, Enfermagem e Pedagogia, apenas com os alunos dos primeiros e últimos anos de cada curso estudado. Foi utilizada a amostragem aleatória, usando-se $20 \%$ da população. Segundo Stevenson (1981: 168), a Amostragem Aleatória Estratificada pressupõe a divisão da população em subgrupos (estratos) de itens secundários, procedendo-se então a amostragem em cada subgrupo. A lógica do processo é que, dispondo os itens da população em subgrupos, a variabilidade é menor que a da população global, o que leva a necessidade de um menor tamanho da amostra.

Os 168 estudantes da amostra que serviram de base a esta pesquisa, apresentam as seguintes características: 
DISTRIBUIÇÃO DA AMOSTRA DOS ALUNOS DOS CURSOS DE PEDAGOGIA, DIREITO E ENFERMAGEM DA UNIVERSIDADE REGIONAL DO CARIRI, SEGUNDO A REPRESENTATIVIDADE, RELATIVA AO NÚMERO DE ALUNOS POR CURSO.

\begin{tabular}{l|c|c|c}
\hline \multicolumn{1}{c|}{ Curso } & $\begin{array}{c}\text { População } \\
\text { Em 2013.1 }\end{array}$ & $\begin{array}{c}\text { Tamanho da } \\
\text { Amostra }\end{array}$ & $\begin{array}{c}\text { Representatividade da } \\
\text { amostra, relativa ao número } \\
\text { de alunos de cada curso (\%) }\end{array}$ \\
\hline Direito & 295 & 70 & 23,72 \\
\hline Enfermagem & 115 & 32 & 27,82 \\
\hline Pedagogia & 239 & 66 & 27,61 \\
\hline Total................... & 649 & 168 & - \\
\hline
\end{tabular}

Fonte: Departamento de Ensino de Graduação - DEG/ PROGRAD/URCA/2013.1.

Segundo a Tabela 1, a amostra é representativa da população da qual procede. A população em 2013.1, objeto do presente estudo, representa a totalidade dos estudantes matriculados nos dois primeiros e nos dois últimos semestres de cada curso, nos turnos existentes.

A população apresentou a seguinte representatividade: $45,46 \%$ de alunos do curso de Direito, 17,72\% de alunos do curso de Enfermagem e 36,82\% de alunos do curso de Pedagogia.

Em relação ao sexo dos participantes, a amostra se comportou da seguinte forma:

TABELA 2 DISTRIBUIÇÃO DA AMOSTRA DOS ALUNOS DOS CURSOS DE PEDAGOGIA, DIREITO E ENFERMAGEM DA UNIVERSIDADE REGIONAL DO CARIRI, POR SEXO.

\begin{tabular}{l|c|c|c|c|c}
\hline Sexo & Enfermagem & Pedagogia & Direito & Total & $(\boldsymbol{\%})$ \\
\hline Masculino & 04 & 08 & 44 & 56 & 33.33 \\
Feminino & 28 & 58 & 26 & 112 & 66.67 \\
Total..... & 32 & 66 & 70 & 168 & 100.00 \\
\hline
\end{tabular}

Fonte: Departamento de Ensino de Graduação - DEG/ PROGRAD/URCA/2013.1

Na sequência, o percentual da amostra em relação ao tempo de curso.

TABELA 3 DISTRIBUIÇÃO DA AMOSTRA DOS ALUNOS DOS CURSOS DE PEDAGOGIA, DIREITO E ENFERMAGEM DA UNIVERSIDADE REGIONAL DO CARIRI, SEGUNDO O TEMPO DE CURSO.

\begin{tabular}{|l|c|c|c|c|c|}
\hline $\begin{array}{l}\text { Tempo de } \\
\text { Curso }\end{array}$ & $\begin{array}{c}\text { Enfermage } \\
\text { m }\end{array}$ & Pedagogia & Direito & Total & $(\boldsymbol{\%})$ \\
\hline Primeiro ano & 18 & 32 & 40 & 90 & 53.58 \\
Último ano & 14 & 34 & 30 & 78 & 46.42 \\
Total & 32 & 66 & 70 & 168 & 100.00 \\
\hline
\end{tabular}

FONTE: Departamento de Ensino de Graduação - DEG/ PROGRAD/URCA/2013.1 
Com relação ao tempo de curso, 53,58\% dos entrevistados cursavam o primeiro ano dos seus respectivos cursos, enquanto $46,42 \%$ estavam em vias de conclusão, isto é, cursando o último ano dos seus respectivos cursos.

\section{Caracterização do campo}

O presente estudo realizou-se na Universidade Regional do Cariri - URCA, localizada no município do Crato, cidade localizada ao sul do Ceará. Trata-se de uma Universidade Pública Estadual, criada em 1986, que atende a região do Cariri, num raio de aproximadamente trezentos quilômetros. Esta pesquisa foi realizada no período de Abril a Junho do ano 2013.

As informações foram colhidas à partir de revisão bibliográfica e de questionário utilizado para avaliação das necessidades de orientação dos alunos, especialmente desenvolvido para este fim.

\section{Resultados e discussão}

\section{Priorização das necessidades de orientação}

Dentre as dez necessidades prioritárias citadas pelos alunos dos três cursos, 60\% são necessidades de orientação profissional e $40 \%$ dizem respeito a necessidades acadêmicas. Incrementar competências e conhecimentos profissionais e obter informações gerais sobre os serviços acadêmicos são as principais necessidades citadas pelos alunos.

TABELA 4 -DISTRIBUIÇÃO DAS NECESSIDADES CITADAS PELOS ALUNOS DOS CURSOS DE DIREITO, ENFERMAGEM E PEDAGOGIA DA UNIVERSIDADE REGIONAL DO CARIRI-URCA, EM RESPOSTA A PERGUNTA: NECESSITAS OU TENS NECESSITADO DE AJUDA NA SEGUINTE ÁREA?

\begin{tabular}{|c|c|}
\hline $\begin{array}{c}\text { RESULTADO DAS NECESSIDADES MAIS CITADAS } \\
\text { PELOS ALUNOS DOS TRES CURSOS (DIREITO, ENFERMAGEM E } \\
\text { PEDAGOGIA) }\end{array}$ & $(\%)$ \\
\hline 107. Incrementar competências e conhecimentos profissionais & 92.86 \\
\hline 29. Obter informações gerais sobre os serviços acadêmicos & 91.67 \\
\hline $\begin{array}{l}\text { 122. Obter mais informações sobre as possíveis saídas profissionais, específicas } \\
\text { do seu curso ou especialidade }\end{array}$ & 91.66 \\
\hline 17. Encontrar apoio de um professor orientador & 91.09 \\
\hline 119. Obter informação atualizada sobre o mercado de trabalho & 91.07 \\
\hline 116. Conhecer os serviços profissionais que a Universidade ofe & 90.48 \\
\hline
\end{tabular}


101. Adequar os estudos a suas atividades, interesses e motivação profissionais

11. Escolher uma especialidade a seguir, de acordo com o curso que escolheu

113. Dispor de um serviço de colocação profissional ou de emprego

35. Conhecer os modos de participação dos alunos, em organismos de poder decisório na Universidade

Em termos da priorização por curso, os alunos do curso de direito sentem mais necessidades profissionais. Estas representam $60 \%$ das dez necessidades mais citadas por eles, destas $40 \%$ são necessidades acadêmicas.

Também entre os alunos do curso de pedagogia, 60\% das dez suas necessidades prioritárias são necessidades de orientação profissional. Entre os alunos do curso de enfermagem, 40\% das dez necessidades prioritárias são referentes a orientação profissional, $40 \%$ são necessidades de orientação acadêmica e $20 \%$ são necessidades de orientação assistencial e/ou de saúde.

Com relação a eleição de prioridades por gênero, tanto os homens quanto as mulheres apresentaram resultados semelhantes. Entre as 10 necessidades citadas como prioritárias, $60 \%$ são relacionadas a orientação profissional e $40 \%$ são necessidades acadêmicas.

Quanto ao tempo de curso, observou-se que os alunos do último ano dos cursos, ainda não tiveram atendidas suas necessidades de orientação acadêmica. Das dez necessidades prioritárias desses alunos, $40 \%$ são necessidades de orientação acadêmica e $60 \%$ são necessidades de orientação profissional. Os alunos dos primeiros anos dos cursos estudados, se revelaram em média, mais interessados em orientação acadêmica (Tabela 57), porém, elegeram a orientação profissional, entre as dez mais imediatas.

Sobre a importância atribuída as necessidades citadas pelos sujeitos, observe-se a tabela a seguir.

TABELA 5 DISTRIBUIÇÃO DA PRIORIZAÇÃO DA IMPORTÂNCIA ATRIBUÍdA A SATISFAÇÃO DAS NECESSIDADES CITADAS PELOS ALUNOS DOS CURSOS DE DIREITO, ENFERMAGEM E PEDAGOGIA DA UNIVERSIDADE REGIONAL DO CARIRI - URCA, EM RESPOSTA A PERGUNTA: QUE IMPORTÂNCIA ACREDITAS TER EM SATISFAZER NECESSIDADE?

\begin{tabular}{l|c}
\hline \multicolumn{1}{c|}{ AS 10 NECESSIDADES TIDAS COMO MUITO IMPORTANTES, } & (\%) \\
\hline NA OPINIÃO DOS ALUNOS, DE UMA FORMA GERAL & 78.57 \\
\hline $\begin{array}{l}\text { 119. Obter informações atualizadas sobre o mercado de trabalho } \\
\text { curso }\end{array}$ & 77.38 \\
\hline \begin{tabular}{l} 
113. Dispor de um serviço de colocação profissional ou de emprego \\
\hline 74. Tomar consciência do seu estado de saúde geral
\end{tabular} & 75.59 \\
\hline
\end{tabular}




\begin{tabular}{l|l} 
77. Conseguir assistência médica satisfatória & 73.21 \\
\hline 101. Adequar os estudos a suas atividades, interesses e motivação profissionais & 72.62 \\
\hline 128. Efetuar adequadamente seu processo de transição Universidade-Trabalho & 72.62 \\
\hline 107. Incrementar competências e conhecimentos profissionais & 72.02 \\
\hline 125. Aprender a solicitar emprego & 72.02 \\
\hline 116. Conhecer os serviços profissionais que a Universidade oferece & 70.83 \\
\hline
\end{tabular}

Crato-CE, 2013

Dentre as dez necessidades tidas como muito importantes, relativamente aos três cursos, é interessante observar que oito (80\%) são necessidades de orientação profissional; e duas (20\%) são necessidades de orientação assistencial e/ou de saúde. Esta informação vem a confirmar a carência de orientação profissional sentida pelos estudantes.

Com relação a dificuldade em encontrar certo tipo de serviço, foi grande o número de alunos que relataram não saberem como obter informações em caso de necessidade. A dificuldade mostrou-se maior quando estas informações são de natureza profissional. Informações como: Conhecer as possibilidades que se oferecem de estudos no estrangeiro ou em outras universidades $(88,69 \%)$; Conhecer os serviços profissionais que a universidade oferece $(88,10)$; Conhecer atividades que a Universidade oferece para o tempo livre (87,50\%); Dispor de um serviço de colocação profissional ou de emprego (82,74\%); Informar-se sobre ajudas econômicas $(82,74 \%)$ ou Obter um bom serviço de restaurante universitário $(82,14 \%)$, que a primeira vista podem parecer simples, representaram uma dificuldade para um número significativo de alunos.

TABELA 6 DISTRIBUIÇÃO DA DEMANDA MÉDIA DAS NECESSIDADES DE ORIENTAÇÃ̃O ALUNOS DA UNIVERSIDADE REGIONAL DO CARIRI (URCA), POR CURSO.

\begin{tabular}{l|c|c|c}
\hline \multicolumn{1}{c|}{ TIPO DE NECESSIDADE } & DIREITO & ENFERMAGEM & PEDAGOGIA \\
\hline Orientação Acadêmica & 74,28 & 78,62 & 85,90 \\
Orientação Pessoal & 56,57 & 71,87 & 71,21 \\
Orientação Assistencial e/ou de Saúde & 59,42 & 71,87 & 73,03 \\
Orientação Profissional & 84,00 & 85,94 & 90,30 \\
\hline
\end{tabular}

Crato-CE, 2013

Conforme os dados obtidos, existe uma grande demanda por orientação em todas as dimensões (acadêmica, pessoal, assistencial e/ou de saúde e profissional). Os alunos de pedagogia 
demonstraram maior demanda, praticamente, em todas as dimensões, sendo a orientação profissional a mais carente de informações, com 90,30\% das necessidades, seguida da necessidade de orientação acadêmica, com 85,90\% de alunos necessitados de informações. Este resultado tende a se repetir com os alunos de enfermagem, quando $85,94 \%$ demandam por orientação profissional e 78,62\% demandam por orientação acadêmica, e com os alunos do curso de direito, onde $84 \%$ demandam por orientação profissional e $74,28 \%$ demandam por orientação acadêmica.

TABELA 7 DISTRIBUIÇÃO DA DEMANDA MÉDIA DAS NECESSIDADES DE ORIENTAÇÃ̃O DOS ALUNOS DA UNIVERSIDADE REGIONAL DO CARIRI - URCA, POR SEXO.

\begin{tabular}{l|c|c}
\hline \multicolumn{1}{c|}{ TIPO DE NECESSIDADE } & MASCULINO & FEMININO \\
\hline Orientação Acadêmica & 73,05 & 81,81 \\
Orientação Pessoal & 58,29 & 69,22 \\
Orientação Assistencial e/ou de Saúde & 59,62 & 72,17 \\
Orientação Profissional & 83,02 & 89,82 \\
\hline
\end{tabular}

Crato-CE, 2013

A pesquisa demonstrou serem as mulheres as mais demandantes por orientação, em todas as dimensões estudadas.

TABELA 8 DISTRIBUIÇÃO DA DEMANDA MÉDIA DAS NECESSIDADES DE ORIENTAÇÃ̃O DOS ALUNOS DA UNIVERSIDADE REGIONAL DO CARIRI - URCA, POR ANO DE CURSO.

\begin{tabular}{l|c|c}
\hline \multicolumn{1}{c|}{ TIPO DE NECESSIDADE } & PRIMEIROS ANOS & ÚLTIMOS ANOS \\
\hline Orientação Acadêmica & 82,91 & 77,07 \\
Orientação Pessoal & 68,29 & 64,61 \\
Orientação Assistencial e/ou de Saúde & 70,25 & 66,80 \\
Orientação Profissional & 78,33 & 85,13 \\
\hline
\end{tabular}

Crato-CE, 2013

De acordo com os dados da tabela acima, os alunos dos primeiros anos de curso demandam, prioritariamente, por orientação acadêmica $(82,91 \%)$, seguida das orientações profissional (78,33\%), assistencial e/ou de saúde (70,25\%) e pessoal $(68,29 \%)$, enquanto que os alunos dos últimos 
anos de curso demandam mais por uma orientação profissional $(85,13 \%)$, seguida das orientações acadêmica $(77,07 \%)$, assistencial e/ou de saúde $(66,80 \%)$ e pessoal $(64,61 \%)$.

TABELA 9 DISTRIBUIÇÃO DA DEMANDA DA IMPORTÂNCIA ATRIBUÍDA ÀS NECESSIDADES, DE ORIENTAÇÃO DOS ALUNOS DE DIREITO, ENFERMAGEM E PEDAGOGIA DA URCA.

\begin{tabular}{l|c|c|c|c}
\hline \multicolumn{1}{|c|}{ TIPO DE NECESSIDADE } & $\begin{array}{c}\mathbf{1} \\
\text { MUITO } \\
\text { IMPORTANTE } \\
(\%)\end{array}$ & $\begin{array}{c}\mathbf{2} \\
\text { IMPORTANTE } \\
(\%)\end{array}$ & $\begin{array}{c}\mathbf{3} \\
\text { POUCO } \\
\text { IMPORTANTE } \\
(\%)\end{array}$ & $\begin{array}{c}\mathbf{4} \\
\text { NADA } \\
\text { IMPORTANTE } \\
(\%)\end{array}$ \\
\hline Orientação Acadêmica & 50,76 & 36,10 & 8,80 & 4,34 \\
Orientação Pessoal & 53,95 & 32,67 & 7,31 & 6,07 \\
$\begin{array}{l}\text { Orientação Assistencial e/ou de } \\
\text { Saúde }\end{array}$ & 55,76 & 30,68 & 7,56 & 6,00 \\
Orientação Profissional & 71,36 & 23,03 & 3,42 & 2,19 \\
\hline
\end{tabular}

Crato-CE, 2013

Dentre as dimensões da orientação apresentadas aos estudantes como opção, foram mais significativas em importância as necessidades relacionadas com a orientação profissional, onde $71,36 \%$ dos alunos a consideraram muito importante. Este percentual se eleva consideravelmente quando consideramos essa necessidade como importante ou muito importante (94,39\%). Há um contingente de $3,42 \%$ dos alunos que consideram essa dimensão como pouco importante e $2,19 \%$ a consideram nada importante. As necessidades relacionadas a assistência social e de saúde, também foram consideradas muito importantes por $55,76 \%$ do alunato, de uma maneira geral. Há $6,0 \%$ dos alunos que consideram essa dimensão nada importante e apenas 7,56\% a consideram pouco importante. Quando consideramos a junção das valorações muito importante ou importante, a dimensão acadêmica também se revela bastante significativa (86,76\%). Há porém 4,34\% dos alunos que consideram pouco importantes as necessidades desta dimensão e 8,80 a consideram pouco importantes de uma maneira geral.

Também pode-se observar que a orientação pessoal foi citada como muito importante por $53,76 \%$ dos alunos de uma forma geral. Quando levamos em conta essa necessidade como importante ou muito importante, este percentual se eleva para $86,62 \%$, revelando o grau de importância atribuída também a esta dimensão. Deve-se levar em conta que 7,31\% dos alunos considera essa dimensão pouco importante e $6,07 \%$ a considera nada importante. 
DISTRIBUIÇÃO DA DEMANDA DOS ALUNOS DA UNIVERSIDADE REGIONAL DO CARIRI - URCA, DOS CURSOS DE DIREITO, ENFERMAGEM E PEDAGOGIA, EM SABER OU NÃO COMO ENCON-TRAR A INFORMAÇÃO REFERENTE AO TIPO DE ORIENTAÇÃO DE QUE NECESSITAM.

\begin{tabular}{l|c|c}
\multicolumn{1}{c|}{ TIPO DE NECESSIDADE } & $\begin{array}{c}\text { Sabe Onde Obter Informação } \\
\text { Nesta Dimensão } \\
(\boldsymbol{\%})\end{array}$ & $\begin{array}{c}\text { Não Sabe Onde Obter } \\
\text { Informação Nesta Dimensão } \\
(\boldsymbol{\%})\end{array}$ \\
\hline Orientação Acadêmica & 31,61 & 68,39 \\
Orientação Pessoal & 44,65 & 55,35 \\
$\begin{array}{l}\text { Orientação Assistencial e/ou de } \\
\text { Saúde }\end{array}$ & 37,17 & 65,83 \\
Orientação Profissional & 21,08 & 78,92 \\
\hline
\end{tabular}

Crato-CE, 2013

É grande o percentual de estudantes que relatam não saberem onde informar-se quando necessitam de orientação nas diversas dimensões propostas nesta pesquisa. A dificuldade de obter informação é maior na orientação profissional, onde $78,92 \%$ do alunato relatou não saber onde informar-se em caso de necessidade. Também é grande o número de alunos que não sabem como conseguir informações acadêmicas (68,39\% dos entrevistados). As dimensões assistencial ou de saúde e a pessoal, foram as menos demandadas, com $65,83 \%$ e $55,53 \%$ das respostas, respectivamente.

\section{Considerações Finais}

Os resultados desta pesquisa confirmam e ampliam os descobrimentos de outras investigações sobre necessidades dos estudantes universitários em matéria de orientação, que nos serviram de referência (Castellano,1995, Campoy e Pantoja,2000). Assim apreciamos de forma global, como os alunos necessitam de ajuda nas diversas áreas investigadas (tabela 58), especialmente nas relacionadas com a Orientação Profissional, com uma demanda média de $86,84 \%$ do alunato, seguida da necessidade de Orientação Acadêmica com 79,64\%.

Não menos preocupados estão os alunos com a ajuda relacionada a Orientação Pessoal $(65,23 \%)$ ou com Orientação Assistencial ou de Saúde $(67,44 \%)$. Uma prova evidente de que nestes temas, não menos importantes que os anteriores, eles não têm uma preparação suficiente para resolver seus problemas, sem necessitar de ajuda complementar.

Os dados resultantes da presente pesquisa nos levam a crer que, uma média de 74,78\% dos estudantes da Universidade Regional do Cariri - URCA, possui algum tipo de necessidade. 
Em relação a importância dos distintos fatores, esta se concentra na necessidade de informação profissional, onde $94,39 \%$ dos entrevistados, a consideram muito importante $(71,36 \%)$ ou importante $(23,03 \%)$. Apenas uma pequena porcentagem dos alunos $(5,61 \%)$, acredita que este tipo de orientação é pouco importante $(3,42 \%)$ ou nada importante $(2,19 \%)$. Uma prova de que de que, aparentemente, este é o principal interesse do alunato desta universidade.

As principais dificuldades que encontraram os alunos em resolverem os seus problemas, concentraram-se, nos serviços de orientação profissional, onde 78,92\% informam que não saberiam como obter informações relacionadas, em caso de necessidade. Segue-se as necessidades de orientação acadêmicas, onde $68,39 \%$ dos alunos, não saberiam como informar-se , em caso de necessidade nesta dimensão (tabela 60).

Das reflexões anteriores, obtemos a conclusão geral de que os estudantes da Universidade Regional do Cariri, manifestam uma demanda maior no campo da Orientação Profissional. Esta necessidade não está sendo satisfeita durante o período universitário, o que tenderá a provocar inquietudes sobre o futuro, quando do término dos estudos universitários do aluno.

Com relação a necessidade de Orientação Acadêmica, pelo menos $(86,86 \%)$ a consideram como muito importante $(50,76 \%)$ ou importante $(36,10 \%)$, enquanto $(13,14 \%)$ a consideram pouco importante $(8,80 \%)$ ou nada importante $(4,34 \%)$. Trata-se pois de uma constatação positiva, no sentido de tomarmos consciência da necessidade de um Serviço de Orientação, que atenda a uma demanda majoritária, do alunato da URCA.

Conclusões que consideramos mais relevantes nesta investigação:

1. Existe uma insatisfação por parte dos estudantes com relação a pouca orientação recebida;

2. As maiores necessidades de orientação dos estudantes encontram-se nas dimensões Profissional e Acadêmica;

3. As necessidades diferem tanto no início da carreira, como no final dos estudos;

4. As saídas profissionais e o incremento de competências e conhecimento profissionais são as necessidades de orientação mais fortemente demandadas pelo alunato;

5. As mulheres tem mais necessidades de orientação, lhe conferem maior importância e informaram maiores dificuldades em obter os serviços de que necessitam;

6. Os resultados desta pesquisa indicam claramente a existência de uma demanda pela criação de um serviço de orientação universitária.

Acreditamos que os estudantes da Universidade Regional do Cariri, objeto deste estudo, questionaram indiretamente a educação que lhes é oferecida. Estamos convencidos de que a resposta a essas necessidades passa pelo planejamento e desenvolvimento de programas de orientação educativa e profissional. Esta última enfatiza a importância da educação para o trabalho, através da inclusão nos 
programas, de objetivos relacionados com o desenvolvimento profissional, para que se aproveite ao máximo os recursos humanos egressos da Universidade. Esta pesquisa demonstrou a urgência da implantação de um serviço de orientação educativa que promova, dentre outras coisas, uma adaptação curricular às necessidades atuais de demanda das empresas e do desenvolvimento social.

A qualidade da educação superior, dentro de uma perspectiva orientadora, não se identifica somente com uma melhor preparação intelectual e técnica dos futuros graduados, mas com o favorecimento do processo de amadurecimento pessoal, que permita tomadas de decisões mais congruentes; capacitação para enfrentar situações problemáticas adequadamente, bem como o desenvolvimento de uma personalidade equilibrada, que permita atuar com mais eficácia na sociedade e nas relações interpessoais. Estes são fatores suficientes para justificar uma intervenção orientadora nesta direção.

Acreditamos que este estudo não será suficiente para dirimir todas as dúvidas com relação à temática abordada, questões como: a) que intervenção orientadora deve ser estabelecida no âmbito universitário?; b) basta levar a cabo um serviço de orientação dirigido para universitários?; c) em que medida o professorado universitário irá assumir sua função tutorial de acordo com o planejamento de uma orientação universitária?; d) que desenvolvimento profissional haveremos de propiciar aos professores para favorecer a consecução dos objetivos de orientação dos estudantes universitários?,provavelmente ainda serão temas, objetos de futuros trabalhos investigativos.

\section{Referências}

ALMEIDA, Núbia Ferreira (Org). Como Escrever Uma Monografia: Manual Informativo. Crato: Universidade Regional do Cariri - URCA/BNB, 1999.

ALVAREZ, M. Orientación Vocacional. Projeto Docente, Universidad de Barcelona, 1992.

ALVAREZ, M. y FERNANDEZ, R. Programa de Orientación de Estudios y Vocacioonal al Término de La Escolaridad Obrigatória, Barcelona, PPU, 1989.

ALVAREZ ROJO, V. Tengo que Decidirme, Servilla: Alfar, 1991.

ALVARES ROJO, V. (et al). Metodologia de la Orientación Educativa, Sevilla: Alfar, 1987.

ANDERSON, S. B. y BALL, S. The Profession and Practice of Program Evaluacion, San Francisco: Jossey-Bass, 1978.

APODACE, P. e LOSATE, C. Calidade en La Universidad: Orientación e Evaluación, Barcelona: Estel, 1997. 
ALZINA, Rafael Bisquerra (Org). Modelos de Orientación e Intervención Psicopedagógica, Barcelona: Praxis, 1998.

BISQUERRA, R. Orientación PsicopedagógicaPara La Prevención Y el Desarrollo. Barcelona: Boixareu Universitaria, 1991.

BRASIL, Leis e Decretos. Diretrizes e Bases da Educação Nacional: LDB N. ${ }^{\circ}$ 5.692/ 71 e LDB n. ${ }^{\circ}$ 9.394/96.

BRITO, Ana Rosa Peixoto de. L.D.B. da "Conciliação" Possível a Lei "Proclamada". Coletânea elaborada pela coordenadora do Ensino Técnico da Secretaria do Estado do Pará: sd.

CAMPOY, Tomás J. e PANTOJA, Antônio. La Orientación en La Universidad de Jaén. Revista RECP, vol 11, N. ${ }^{\circ} 19,1^{\circ}$ semestre, 2000, p.77-106.

CARKUFF, R. and BERENSON, B. Beyond couseling and therapy. New York: Holt R. e Winston Co., 1967.

CASTEllano, F. M. Análisis de las Necessidades de los Estudiantes de la Universidad de Granada en Orientación Educativa. Tesis Doctoral. Universidade de Granada, 1993.

La Orientación Educativa en la Universidad de Granada: Evaluaçión de las necessidades. Universidade de Granada, 1995.

. Evoluçión de Las Necessidades de Orientación Educativa en Universitários de La Faculdade de CC. EE. de Granada. Revista de Orientacion Educativa, vol. 5, № 8, Granada, 1994.

CIRIGLIANO, G. Filosofia de la Educación. Buenos Aires: Humanitas, 1972.

COSTA, Ana R. F. (et al). Orientações Metodológicas para Produção de Trabalhos Acadêmicos. Maceió: EDUFAL, 1997.

DANTAS, Avany L. Metodologia da Pesquisa. João Pessoa: UFPB, 1997.

DELGADO, J. A. Deseño y Evaluación de un Programa de Educación Vocacional al Término de la EGB. Tesis Doctoral. Universidade de Granada, 1993.

ECHEVERRÍA, B. y RODRIGUZ ESPINAR, S. Proyecto de un Centro de Orientación Acadêmica y Prodesional. (I Simpósio Sobre Orientación Académica y Profesional en la Universidad). Barcelona, 1989.

ECHEVERRÍA, B. La Orientación y La Formación Ocupacional. Madrid: VII Jornadas Nacionalesde AEOEP, 1991.

FERRETTI, João Celso. Uma Nova Proposta de Orientação Profissional. 2. ed. São Paulo: Cortez, 1992.

FUNDAÇÃO INSTITUTO DE PESQUISA E INFORMAÇÃO DO CEARÁ - IPLANCE. Anuário Estatístico do Ceará (1998-1999). Disponível em: 〈http://www.iplance.ce.gov.br〉. Acesso em: 05/jan/2002.

GARCÍA HOZ, V. Educación Personalizada. Madrid: CSIC, 1970. 
GERENCER, Pavel e GERENCER, Paul. Educação para o Trabalho. São Paulo: Atlas, 1979. 2 v.

GILL, S. J. y FRUELling, J. A. Needs Assessment and The Desing of Service Delivery Systems. Journal os College Student Personnel, 1979.

GONÇALVES, Maria Domingas. Escolha a sua profissão. Rio de Janeiro: Edições de Ouro/Tecnoprint, 1980.

GONDIM, Linda M. P. (Org). Pesquisa em Ciências Sociais: O Projeto da Dissertação de Mestrado. Fortaleza: EUFC, 1999.

GRINSPEEN, Miriam. P. Zippim. (et al). A Prática dos Orientadores Educacionais. Cortez, 1994.

HAMEZ, Suely. Segurando a barra dos pais. Guia do Estudante. São Paulo: Abril Cultural, 1997.

HANNOUN, Hubert. Educação: Certezas e Apostas. São Paulo: UNESP, 1998.

HERR, E. R. Perspective on Career Guidance in the 21 st Century: Educacional and Vocacional Guidance, 1997.

HERRERA, L. La Orientación Educativa y Vocacional. México: Porrúa, 1976.

HIGGINS, E. B., Comunity College Couselling Centers: Structure and Focus. Comunity College Review, 1981.

HOYT, K., EVANS, R., MACKON, E. y MAGNUM, G. Career Educación: What it is to do it. Salt Lake City. Uyah: Olimpus Publishing Company, 1972.

INSTITUTO BRASILEIRO DE GEOGRAFIA E ESTATÍSTICA - IBGE. Cidades: 2000. Disponível em 〈http://www.ibge.gov.br/cidadesat/default.php>. Acesso em: 05/Jan/2002.

JASPERS, K. La Filosofia desde el punto de vista de la Existência. 1962.

JONES, A. Principles of Guidance. Nova York: McGraw Hill Book Co., 1951.

KANN, U. La Orientacion Vocacional y el mercado de trabajo. Oerspectivas, 1988.

KATZ, M. R. "Informatización de la OrientaciónVocacional y Sistemas de Información sobre Empleo". Perspectivas, 68, 545-558, 1988.

KAUFMAN, R. Identifyng and Solving Problems: A System Approach, San Diego, Califórnia: University Associates, 1982.

KLINE, Paul. Psicologia da Orientação Vocacional. (Trad.) Álvaro Cabral. RJ:Zahar, 1977 @ 1975.

LAS, S. Orientação Universitária: Do Ensino Secundário a Universidade. Lleida: Universidade de Lleida, 1995

LÁZARO, A. y ASENSI, J. Manual de Orientación Escolar y Tutoría. Madrid: Narcea, 1987. 
LOBATO, C. y Muñoz, M. Diagnóstico de Necessidades de Orientación Universitária. XV Congresso Internacional de Recursos Humanos. Orientación y Mercado Laboral, Madrid, 1994.

LOFREDDI, Laís Esteves. Encontros e Desencontros na Escola: O Papel do Orientador Educacional. Rio de Janeiro: Francisco Alves, 1980.

LOURENÇO FILHO, M. B. Orientação Educacional. Revista Brasileira de Estudos Pedagógicos, 1945.

LUCCHIARI, Dulce Helena Penna Soares (org); LISBOA, Marilu Diez; PRADO FILHO, Kleber. Pensando e Vivendo a Orientação Profissional. São Paulo: Summus, 1993.

LUIPJEN, W. Introdução à Fenomenologia Existencial. Trad. Carlos Lopes de Mattos. São Paulo: EPU/EDUSP, 1973.

LÜCK, Heloísa. Planejamento em Orientação Educacional. Petrópolis: Vozes, 1991.

MAIA, Pe. Pedro Américo, S.J. Profissões e Vida: Acerte na Escolha de seu Futuro. Cadernos de Formação e Cultura, n. ${ }^{\circ}$ 29. BH: Grupo Gente Nova, 1968.

MASLOW, Abraham H. Introdução à Psicologia do Ser. Trad. Álvaro Cabral. 2. ed. Rio de Janeiro: Eldorado, s.d.

MARTINS, Carlos Roberto. Psicologia do Comportamento Vocacional: Contribuições para o Estudo da Psicologia do Comportamento Vocacional. São Paulo: EPU/EDUSP, 1978. ( 1978.

MARTINS, José do Prado. Princípios e Métodos de Orientação Educacional. 2. ed. São Paulo: Atlas, 1987.

MAY, Rollo. A Descoberta do Ser. Trad. Claudio G. Somognyi. Rio de Janeiro: Rocco, 1993. (C) 1983.

O Homem à Procura de Si Mesmo. Trad. Áurea Brito Weisseberg.

19. ed. Rio de Janeiro: Vozes, 1993. (C) 1953.

Psicologia e Dilema Humano. Trad. Álvaro Cabral. Rio de Janeiro: Zahar, 1977. @ 1967.

MELO, Sonia Maria Martins. Orientação Educacional: Do Consenso ao Conflito. São Paulo: Papirus, 1994.

MIRA y LOÓPEZ, E. Manual de Orientação Profissional. Buenos Aires: Editorial Kapelugz, 1947.

NÉRECI, Imídeo Giuseppe. Introdução a Orientação Educacional. 5. ed. São Paulo: Atlas, 1996.

NISKER, Arnaldo. A Nova Escola. As Leis: 4024/61, 5.692/71 e 7044/88. Rio de Janeiro: Nova Fronteira, 1986.

PENNA, Antonio Gomes. Percepção e Realidade: Introdução ao Estudo da Atividade Perceptiva. 2. ed. Rio de Janeiro: Fundo de Cultura, 1973. 
PENTEADO, Wilma Milan Alves. (org.). Fundamentos de Orientação Educacional. São Paulo: EPU, 1976.

Edicaon, 1980.

- Wilma Milan Alves. Orientação Educacional: Fundamentos Legais. São Paulo:

PÉREZ, C., RIVAS, F. e ROCABERT, E. Sistema de assessoramiento vocacional para cegos. Revista de psicologia general e aplicada. Granada: out/1992.

PIMENTA, Selma Garrido. Orientação Profissional e Decisão: Estudo Crítico da Situação no Brasil. 5. ed. São Paulo: Loyola, (C) 1979, 1986.

PIMENTEL, Maria da Glória B. O Professor em Construção. São Paulo: Papirus, 1993.

PIMENTEL, Maria da Glória B. e SIGRIST, Áurea C. Orientação Educacional. São Paulo: Pioneira, 1971.

PINILLOS, J. L. Apuntes en torno a las Humanidades y la Ciencia. Madrid: Arbor, 1951.

REPETTO, E. La Personalización en la Relación Orientadora. Madrid: Miñon, 1977.

RIVAS, F. Psicología Vocacional: Enfoques de asesoramiento. Madrid: Morata, 1988.

RODRIGÈZ MORENO, M. L. Orientación e Intervención Psicopedagógica. Barcelolona: CEAC, 1995.

RODRÍGUEZ ESPINAR, S. Proyeto Docente e Investigador. Universidade de Barcelona, 1985.

ROURERA, R. y OUTROS. Orientación Universitária: Um Projeto para a Universidade de Lleida. XV Congresso Internacional de Recursos Humanos. Orientación y Mercado Laboral. Madrid: 1994.

RUDIO, Franz V. Introdução ao Projeto de Pesquisa Científica. Petrópolis: Vozes, 1978.

SANCHÈZ, S. Dicionario de las Ciencias de la Educación. Madrid: Diagonal-Santillana, 1983.

SANTOS, Oswaldo de Barros. Psicologia Aplicada à Orientação e Seleção Profissional. São Paulo: Pioneira, 1976.

SANZ ORO, R. Metodologia de la Orientación Preventiva. En Álvarèz Rojo, V. Metodologia de la Orientación Educativa, Sevilla, Alfar, 1987.

Evaluación de Programas en Orientación Educativa. Madrid: 1990.

SAULNIER-CAZALS, Josette. Educación en La Orientación Universitária. s.l., s.d.

SAVIANI, Demerval. A Nova Lei da Educação: Trajetórias, Limites e Perspectivas. 2. ed. Campinas, SP: Autores Associados, 1997.

SCHEEFFER, Ruth. Aconselhamento Psicológico: Teoria e Prática. 6. ed. São Paulo: Atlas, 1977. . Teorias de Aconselhamento. São Paulo: Atlas, 1979. 
SEVERIANO FILHO, Cosmo. Metodologia de Pesquisa Orientada à Elaboração do Trabalho Monográfico. João Pessoa: UFPB, 1998.

STEVENSON, Willian J. Estatística Aplicada à Administração. (Trad.) Alfredo Alves de Farias. São Paulo: Harper \& Row do Brasil, 1981.

SUPER, D. Transition in the USA: From Vocational Guidance to Couselling Psychology. Bulletin de l'Association Internationale de Psychotechnique, 1953.

SUPER, Donald Edwine e BOHN JR, Martim. Psicologia Ocupacional, (Trad.) Esdras do Nascimento e Jair Ferreira dos Santos do título original Occupational Psychology, (C) 1970. São Paulo: Atlas, 1980. () 1980.

SUPER, D. E. "A Development Theory: Implementing a Self Concept". In.: Monttross D. H. y Shinkman (Ed). Career Development in 1980: Theory na Pratice. Sprinfield: Thomas, 1981.

TEJEDOR, F. J. Perspectiva Metodológica del Diagnóstico y Evaluación de Necessidades en el Ámbito Educativo. RIE, 1990.

TYLER, L. La Función del Orientador. México: Trillas, 1983.

TOFFLER, Alvin. Future shock. New York: Bantam, 1970.

UNIVERSIDADE REGIONAL DO CARIRI - URCA. A Instituição. 2001, Disponível em $<$ http://www.urca.br/institui.html >. Acesso em: 06/Jan/2002.

WATTS, A. G. "La Evolución de la Orientación Vocacional en la Escuela". Perspectivas, 68, 499508, 1988.

WATTS, T. Careerquake. Revista de Orientación y Psicopedagogia. Ano 8, N. ${ }^{\circ}$ 14, p. 217-221, 1997.

WHITAKER, Dulce. A Escolha da Carreira. São Paulo: Moderna. 1985.

ZAMORAnO, P. y OLIVEROS, L. Análisis de Necessidades en los Alumnos de Primer Curso en la Faculdad de Educación. XV Congresso Internacional de Recursos Humanos. Orientación y Mercado Laboral. Madrid: 1994.

Como citar este artigo (Formato ABNT):

BRAGA, I.B.; COELHO, M.N. Necessidades de Orientação Educativa na Universidade Regional do Cariri. Id on Line Revista de Psicologia, Fevereiro de 2015, vol.9, n.25, p. 38-61 ISSN 1981-1189.

Recebido: 10/01/2015

Aceito: 22/01/2015 